\title{
MORC Family CW-Type Zinc Finger Protein 3
}

National Cancer Institute

\section{Source}

National Cancer Institute. MORC Family CW-Type Zinc Finger Protein 3. NCI Thesaurus. Code C113142.

MORC family CW-type zinc finger protein 3 (939 aa, 107 kDa) is encoded by the human MORC3 gene. This protein is involved in both RNA binding and nuclear matrix protein binding. 